\title{
Case Study: Clinical Management of Claustrophobia and Cold Sensitivity towards Operating Room Environment with Preoperative Hypnosis
}

\author{
Dr Anand Chandrasegaran, MBBS, Mmed (Anesthesia) ${ }^{1 *}$
}

${ }^{1}$ Consultant Anesthesiologist \& Critical Care Medicine, Columbia Asia Hospital-Klang, Anesthesiology \& Critical Care Medicine Department, Columbia Asia Hospital-Klang, Selangor, Malaysia

\section{ABSTRACT}

We present a case of utilizing preoperative hypnosis technique in overcoming patient's fear of operating room's (OR) coldness and its small space fear (claustrophobia). In this report, we highlight how these two aspects of an OR environment can prevent patients to be maintained in a constant hypnotic trance state. Operating room can be a claustrophobic experience to some patients. The patient may not have experienced this situation. Therefore, it is important for anesthetist using hypnosis technique to be aware of possible anxiety attack being triggered by claustrophobia or sense of losing control of oneself. It is important to avoid suggestions that can trigger panic attacks, in this case, it was the sensitivity to OR coldness and detailed description of the OR environment.

Keywords: Preoperative hypnosis, Operating room environment, claustrophobia, cold environment

\section{INTRODUCTION}

We present a case of utilizing preoperative hypnosis technique in overcoming patient's fear of operating room's (OR) coldness and its small space fear (claustrophobia). In this report, we highlight how these two aspects of an OR environment can prevent patients to be maintained in a constant hypnotic trance state. After an extensive literature review on any similar case presentation, we found no similar reporting has been made.

\footnotetext{
*Correspondence: mbbsumagmail.com

Dr Anand Chandrasegaran, Hospital Columbia Asia - Klang, PT 71153, Jalan Mahkota 1/KU2, Mutiara Bukit Raja 2,KM 2, Off Jalan Meru,41050 Klang,

Selangor Darul Ehsan, Malaysia, Telephone number: +60 0176502428
}

Received: 06 January 2018 Accepted: 25 January 2018

Sleep and Hypnosis

Journal homepage:

www.sleepandhypnosis.org

ISSN:1302-1192 (Print) 2458-9101 (Online)
There is a substantial amount of evidence that hypnosis represents a useful, additional tool that anesthetists may find valuable in everyday practice (Cyna, Tomkins, Maddock, \& Barker, 2007; Katcher, Segal, \& Beck, 1984). Many anesthetists are using hypnosis to alleviate patient's anxiety at waiting area before wheeling them to the operating room (Saadat et al., 2006). Hypnosis preoperatively is a technique which results in high patient satisfaction and better surgical outcomes (Montgomery, Weltz, Seltz, \& Bovbjerg, 2002).

Purpose of this case report is to highlight the importance of avoiding negative suggestions that may trigger patient's unwanted experiences and emotions associated with it. These suggestions are referring to Operating room's (OR) environment that clinicians may use to describe during the hypnotic induction and maintenance process. However, some of these suggestions may not be appropriate and many clinicians have not 
taken it with much consideration. Two variables in OR environment that should be considered during an initial preoperative interview with patients (for anesthetists this is referring to preoperative assessment review), are: claustrophobia (referring to fear of confined spaces with or without sense of losing own self control) and cold sensitivity (referring to cold environment in OR). Using inappropriate words/suggestions can trigger undesirable experience, and not able to maintain patient in the trance state (relaxed state) leading to failure in maintaining the patient in trance concentration state.

\section{CASE HISTORY}

Mrs. GL is a 28 years old female of Indian origin. She is a person with good educational background and she is a knowledgeable medical staff. She was planned for a surgical procedure (diagnostic arthroscopy of her right knee). She was having the pain in her right knee for almost 4 months after she sustained a fall at her workplace. She is generally an active person. As a result of the injury, she has been having constant pain in her right knee. She consulted an orthopedic surgeon and after MRI investigation of her right knee, a diagnosis of meniscus injury with a patella displacement, of her right knee joint was made. Subsequently, she was planned for an elective surgery for the knee joint.

Anesthesia assessment indicates she is ASA (American Society of Anesthesiologist) Class 1. Good cardiac function, normal general physical examination. She was classified as low-risk anesthesia for low-risk surgery. Mode of anesthesia which was suitable and consented by the patient was general anesthesia. On initial preoperative assessment visit, issues relating to anesthesia care were discussed with the patient. She has some limitation in her right leg flexion and pain on extension. She was on oral non-steroidal anti-inflammatory drugs (NSAIDS) for her analgesia need. On further history taking, there was no significant medical and social history of note. She is not any medications for chronic illness. Her mental state was perfectly normal. She has no psychological history of note. No history of allergy to food or medications. All relevant blood test were within normal parameters limit.

\section{DESCRIPTION OF TREATMENT}

This is the patient's first surgical and anesthesia experience. She was slightly apprehensive about the surgery. As our routine anesthesia approach, this patient was offered a pre-operative hypnosis session, using Erikson's method (Gay, Philippot, \& Luminet, 2002), to reduce her pre-operative anxiety and for general wellbeing motivation. In the preoperative assessment visit, we gathered as much information as possible that can be utilized for the patient's hypnotic trance induction and maintenance process. Written anesthesia and hypnosis consent was obtained after detailed explanation. For this patient, she didn't want any oral anxiolytic agents for her premedication. She was relatively calm and didn't exhibit any signs to suggest anxiety towards the procedure.

On the day of the surgery, the preoperative hypnosis was initiated 30 minutes prior to surgery, which was conducted in the OR waiting area. As our routine practice, suitable patients for preoperative hypnosis will receive only one hypnosis intervention, 30mins before surgery time. The session started with simple eye closure induction with visualization of patient's favourite place (gentle induction with permissive approach). In her case, it was a seaside/beach experience. This was followed by Hartland's progressive muscle relaxation. Hypnosis trance depth moderate level reached, as patient's $\mathrm{hr}$ : 50-60/min with Systolic BP ranges: 110-115mmh, diastolic BP ranges: $70-80 \mathrm{mmHg}$. This was evident, with patient's hypnotic phenomenon manifestations, and deep muscle relaxation: head resting comfortably on the bed, slow and delayed verbal response. The hypnotic suggestion of ego strengthening and general postoperative well being were suggested. Hypnotic suggestions of postoperative successful recovery with rapid healing were given. The suggestion of amnesia also included. We also included suggestions for describing the events that usually take place in $O R$ in relation to preparing the patient for general anesthesia. These included detailed description, of the operating room (OR) environment and placement of monitoring devices on patients, prior to providing anesthesia for the patient. These suggestions were reenforced with supportive statements assuring the patient 
that the doctors and nurse are with her all the time to ensure her safety and well being.

Once patient became relaxed and in a moderate trance state (Shor, 1962)(profound muscle relaxation state where hypnotic phenomenon limb cataplexy was elicited), with stable hemodynamic parameters (blood pressure and heart rate), we wheeled her to the operating table to start the formal anesthesia induction with medications (propofol, fentanyl and gas inhalation: sevoflurane, dosing depending on patient's response). Once anesthesia pharmacological agents used to start anesthesia process, standard anesthesia care will be followed.

\section{RESULTS \& FOLLOW-UPS}

The initial expectation from this was the usual patient comfort and reduction in preoperative anxiety with patient's satisfaction in regards to smooth anesthesia induction. For this patient, it was expected that the standard pre-operative hypnosis would make the patient very relaxed and calm before wheeling into the operating room.

As the patient was already in the deeply relaxed state, decision to wheel patient into the operating table was made. As the patient was being wheeled in, continuous verbal suggestions of relaxation (utilizing distractions to further deepen patient in their hypnotic trance state) were delivered. As patient moving into the OR surgical bed, we were describing the operating environment and noisy distractions in a positive way, to further relax the patient. Suggestion, "you are being wheeled in OR now, but you don't have to worry, as you are in safe hands of doctors and nurses here, you can remain to be relaxed and enjoy your favorite safe place. If you feel cold, it is just the coldness of the operating room, which will be a signal for you to relax more and you could imagine hugging or wrapping yourself in a warm blanket". At this point, a warming mattress device (usual practice for all patients undergoing surgery) will be used to warm patients. We kept positive suggestion, such as "although you are in the operating table, your body needs to be here, but your mind doesn't. You can choose to continue to be safe and at peace at your favourite place." At this juncture, we noticed her facial expression started to changed to more stressed looking, frowning of the forehead, and the patient started to shiver. No anesthesia pharmacological drugs administered here yet. The patient started to breathe heavily and her respiratory rate beginning to increase. Her heart rate on the monitor ranges 90-110/ $\min$.

As suggestions to induce more relaxation and calmness were continued she became more agitated and opened her eyes, looking frightened. The patient was not able to further concentrate on suggestions. Heart rate increased to $100-110 / \mathrm{min}$ with blood pressure ranging: 120 $140 / 90 \mathrm{mmhg}$. This was evident that she was already anxious. The patient was not able to concentrate on further suggestions. A gentle dose of intravenous midazolam $1 \mathrm{mg}$ was given, to calm the patient. However, the patient became more agitated and the patient got up wide awake. She verbalized surgery refusal and wish to cancel the surgery. At this point, we as the medical team in charge of her care decided to postpone the surgery, from a medical ethical perspective. The patient was wheeled out of OR, and back to the ward, awake fully for further review and reassessment.

2 hours later, in the ward, with normal hemodynamic vital signs, the patient was alert. On further questioning, the patient revealed that the night and morning before surgery, she was very anxious and afraid of the surgery. But when the hypnotic relaxation started, the patient said, she was calmer and more relaxed. In fact, she doesn't remember much, except for the feeling of being light and relaxed. Amnesia is a hypnotic phenomenon (Defechereux et al., 1999; Kihlstrom, 1985), which she had exhibited and indicated the adequate hypnotic depth. Patient revealed, as the suggestion of wheeling patient into the OR was made, and OR being cold, she felt a sense of being trapped in small space and losing control. She said, she was getting more distressed, and suddenly her breathing was rapid. Patient and her husband gave an additional history that she has fear of confined spaces. She has a sense of being not in control and extreme fear in small places. This information was not revealed to us at any time during the initial pre-operative visits. Patient 
claims never thought being in OR would trigger this experience for her, despite she being a medical staff. She said she felt a sense of overwhelming panic. We reassess the patient and a new medical consent was obtained from herself and her family members. We agreed to continue with the surgery in the evening, with same anesthesia approach.

This time, similar hypnotic induction was used. The patient became relaxed rapidly. But this time, as we were wheeling patient into $O R$, we only gave positive ego strengthening and well-being suggestions, without mentioning words relating to OR room, coldness, or anything relating to claustrophobia and kept the $O R$ environment as silent as possible, minimizing verbal conversations and sound from monitoring devices. Suggestions for self-control to overcome fear using "dial up/down knob" were included. The patient was not shifted to the OR operating table immediately (as for usual norm practice, patient's would be asked to be on the OR operating bed). This patient was allowed to continue to be in her trance state on her own ward bed. As the patient was already in a deep trance state, this time, we induced patient with intravenous anesthesia method, dose-dependent, as compared to usual technique of co-induction, intravenous and gaseous induction. Our aim here was to prevent excessive activities around the patient in the OR. The patient was successfully anesthetized and surgery proceeded well. Postoperative, patient return to the ward well. Upon asking her experience, on the next postoperative day, the patient has no recollection of events in OR after starting the hypnotic relaxation procedures, and the patient felt more in control this time. She was satisfied with the hypnotic process, and she described being intensely involved with her favourite place.

\section{DISCUSSION}

Hypnosis is an effective adjunctive procedure for a wide variety of surgical patients (Montgomery, Weltz, et al., 2002; Saadat et al., 2006). Hypnosis has proven to reduce post-surgery pain and distress. Initial evidence suggested that the effects of hypnosis were mediated by pre-surgery expectations (Montgomery, Weltz, et al., 2002; Saadat et al., 2006). It is recognized that preoperative anxiety can have adverse effects on the course and outcome of surgery (Markland \& Hardy, 1993). There is a considerable amount of research into the influences of interventions for pre-operative anxiety on a number of post-operative variables (Markland \& Hardy, 1993). This case study gave us an opportunity to look into the effects of patient's perception of Operating Room's environment on the ability to maintain patients in their trance state.

Operating room can be a claustrophobic experience to some patients. The patient may not have experienced this situation. Therefore, it is important for anesthetist using hypnosis technique to be aware of possible anxiety attack being triggered by claustrophobia or sense of losing control of oneself. It is important to avoid suggestions that can trigger panic attacks, in this case, it was the sensitivity to OR coldness and detailed description of the OR environment. In many hypnosis scripts, clinicians using hypnosis often follows a set of hypnotic script or modify according to patient's needs. In this case experience, it shows that it is important to first identify with patients their fear of small, enclosed spaced, and fear of extreme coldness.

Initially, the aim of hypnosis preoperatively, in this case, was to provide the patient a sense of self-confidence and anxiety relieve. However, after patient exhibit, the fear of OR environment, the hypnosis intervention, gave a tool to the patient to self-control her fear. The "dial-up/ down knob" metaphor was used to help patient to visualize them controlling their fear, proved to be a successful approach, because for this patient being in selfcontrol in important. Being able to control and manipulate the degree of her fear in trance state, further enhances her hypnotic experience because the patient believes she still can be in control of herself despite being in a trance state. As for cold OR environment, we avoided mentioning words such as the cold. Instead, we suggested to the patient as follow: " as you are walking down the beach and feeling the slight warmness of the sea water touching your feet, maybe you could imagine holding or putting on a warm scarf around your neck or body. Try to feel the warmth of the scarf." As this suggestion was being 
offered, we applied the warming blanket in OR on the patient. This made the patient warm. No shivering was noted from this patient from the point of the hypnotic induction to anesthesia induction. Avoiding suggestions describing OR environment for this patient was important. This may not be the same for other patients. Therefore, getting a history of patient's prior experience in OR in crucial. The strategy used here was minimizing interruptions to patient's hypnotic experiences and minimizing OR surrounding activities. This was done by not moving the patient to the OR operating table to induce anesthesia (in this case, we anesthetized patient on her ward bed) and controlling OR distractions (conversations amongst medical staff in OR and OR preparations).

Extensive researches have been done on the role of clinical hypnosis in pre-operative setting and benefits of hypnosis for patients (Defechereux et al., 1999; Montgomery, David, Winkel, Silverstein, \& Bovbjerg, 2002). However, limited data is available on hypnotic techniques and suitable scripts available on pre-operative hypnosis. Further studies into hypnotic scripts for trance induction, maintenance and termination scripts, used for pre-operative settings needs, need to be looked into.

\section{References}

Cyna, A. M., Tomkins, D., Maddock, T., \& Barker, D. (2007). Brief hypnosis for severe needle phobia using switch-wire imagery in a 5-year old. Pediatric Anesthesia, 17(8), 800-804. doi:10.1111/j.1460-9592.2007.02224.x

Defechereux, T., Meurisse, M., Hamoir, E., Gollogly, L., Joris, J., \& Faymonville, M. E. (1999). Hypnoanesthesia for endocrine cervical surgery: A statement of practice. Journal of Alternative and Complementary Medicine, 5(6), 509-520. doi:DOI 10.1089/acm.1999.5.509

Gay, M. C., Philippot, P., \& Luminet, O. (2002). Differential effectiveness of psychological interventions for reducing osteoarthritis pain: a comparison of Erickson hypnosis and Jacobson relaxation. European Journal of Pain-London, 6(1), 1-16. doi:10.1053/eujp.2001.0263

Katcher, A., Segal, H., \& Beck, A. (1984). Comparison of Contemplation and Hypnosis for the Reduction of Anxiety and Discomfort during Dental Surgery. American Journal of Clinical Hypnosis, 27(1), 14-21. doi:Doi 10.1080/00029157.1984.10402583

Kihlstrom, J. F. (1985). Posthypnotic Amnesia and the Dissociation of Memory. Psychology of Learning and Motivation, 19, 131178. doi:Doi 10.1016/S0079-7421(08)60526-5
Markland, D., \& Hardy, L. (1993). Anxiety, Relaxation and Anesthesia for Day-Case Surgery. British Journal of Clinical Psychology, 32, 493-504. doi:DOI 10.1111/j.2044-8260.1993.tb01085.x

Montgomery, G. H., David, D., Winkel, G., Silverstein, J. H., \& Bovbjerg, D. H. (2002). The effectiveness of adjunctive hypnosis with surgical patients: A meta-analysis. Anesthesia and Analgesia, 94(6), 1639-1645. doi:Doi 10.1097/00000539200206000-00052

Montgomery, G. H., Weltz, C. R., Seltz, M., \& Bovbjerg, D. H. (2002). Brief presurgery hypnosis reduces distress and pain in excisional breast biopsy patients. International Journal of Clinical and Experimental Hypnosis, 50(1), 17-32. doi:Doi 10.1080/00207140208410088

Saadat, H., Drummond-Lewis, J., Maranets, I., Kaplan, D., Saadat, A., Wang, S. M., \& Kain, Z. N. (2006). Hypnosis reduces preoperative anxiety in adult patients. Anesthesia and Analgesia, 102(5), 1394-1396. doi:10.1213/01. ane.0000204355.36015.54

Shor, R. E. (1962). Three dimensions of hypnotic depth. International Journal of Clinical and Experimental Hypnosis, 10(1), 23-38. 